\title{
Disposición a pagar por vacunas contra el virus papiloma humano en la Región Metropolitana de Santiago de Chile
}

\author{
ARCADIO A. CERDA ${ }^{1, a}$, LEIDY Y. GARCÍA ${ }^{2, b}$, \\ FABIÁN I. GAETE ${ }^{1, \mathrm{c}}$, TOMAS H. PIZARRO ${ }^{1, \mathrm{~d}}$
}

\section{Willingness to pay for human papillomavirus vaccine in Metropolitan Santiago, Chile}

\begin{abstract}
Background: Human papillomavirus (HPV) infection is a risk factor for cervical cancer and can be prevented with the HPV vaccine. Aim: To explore the willingness of parents to pay for HPV vaccine for their offspring. Material and Methods: A survey about the willingness to pay for HPV vaccine was answered by 386 individuals of the highest socioeconomic level who had a daughter aged between 12 and 18 years. The survey included information about the risks of HPV infection. Results: Parents would pay a mean of US\$ 758 for the vaccine. Twenty five percent of parents were not willing to pay for it. If the cost of the vaccine would be reduced by $50 \%$, only $4 \%$ of parents would not pay for it. The willingness to pay is associated with the price of the vaccine, the income level of respondents and the size of the family. Conclusions: Most respondents would pay for HPV vaccine for their daughters, despite the relatively high cost.

(Rev Med Chile 2013; 141: 167-172).
\end{abstract}

Key words: Health care surveys; Papilloma virus vaccines; Socioeconomic factors.

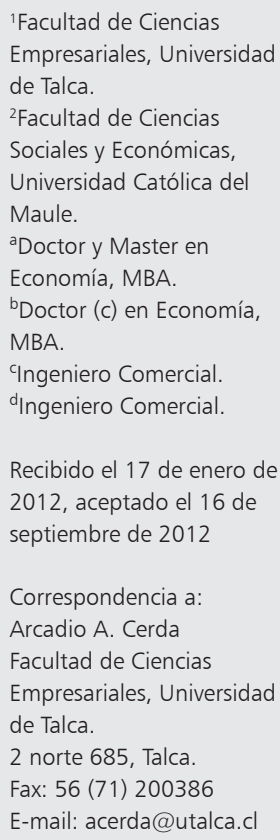

L os costos en términos de vidas y tratamientos generados por virus papiloma humano (VPH) quedan en manifiesto con los cerca de medio millón de casos de mujeres con cáncer cérvico-uterino (CCU) causados por este agente viral a nivel mundial ${ }^{1}$. La distribución mundial del VPH se encuentra principalmente en África y América Latina con los tipos 16, 18, 31, 33, entre otros $^{2}$. El VPH 16 es el más frecuente en el mundo, excepto en Argelia e Indonesia, donde el virus más frecuente es el $18^{3}$.

En Chile mueren alrededor de 750 mujeres anualmente por este tipo de cáncer ${ }^{4}$. La infección causada por el VPH es considerada como enfermedad de transmisión sexual más habitual ${ }^{5}$, de ahí la importancia epidemiológica. Además, Abarca et al, (2008) en la Declaración del Comité Consultivo de Inmunizaciones de la Sociedad Chilena de Infectología respecto a la vacuna anti-VPH mencionan que la infección por este agente viral y el
CCU asociados a los VPH 16 y 18 constituyen un importante problema de salud pública en Chile.

De acuerdo a MINSAL ${ }^{6}$, el principal factor de riesgo de infección por el VPH se determina por la conducta sexual del individuo. Por ello, resulta relevante que los padres tomen conciencia sobre el impacto del VPH, para motivarlos a proteger a sus hijas contra este virus, lo que se puede realizar mediante las vacunas disponibles Gardasil ${ }^{\circledR}$ y Cervarix ${ }^{\circledR 1}$. Adicionalmente, las vacunas contra las infecciones de transmisión sexual son útiles para la reducción de costos de la atención pública de la salud, lo que debiera generar un compromiso compartido entre padres y Estado para solventar dicho gasto.

A nivel de padres, existen escasos estudios que midan la disposición a pagar (DAP) de ellos por vacunas anti VPH. La mayoría de los estudios sólo se focalizan en la actitud o preferencias de los padres por vacunar a sus hijas ${ }^{9-12}$, los que generalmente 
no consideran variables relevantes en el proceso de compra de vacunas, como son los tratamientos alternativos, los precios de las vacunas y el ingreso. De los escasos estudios que consideran la valoración económica, y específicamente DAP contra el VPH, utiliza el método de análisis conjunto ${ }^{13}$.

Otros estudios realizan análisis de costo-efectividad de las vacunas ${ }^{14,15}$, los que encuentran que existe una reducción de costos con el tratamiento preventivo y que las variables que más lo explican son la duración de la protección de la vacuna, el costo de la vacuna y la calidad de vida perdida ajustada por los años, y eran menos sensible a los costos médicos. Uno de los estudios más detallados a nivel nacional es el desarrollado por MINSAL (2011), donde se sistematiza la información sobre CCU en Chile ${ }^{6}$.

En Chile existe escasa información con respecto a la DAP privada por vacunas que reduzcan los riesgos del VPH, por ello el tema de este trabajo es de alta relevancia para el caso chileno, lo que podría reducir una eventual carga financiera al sistema público en el caso que se dispusiera de una aplicación generalizada en el sistema público de salud. Aunque dicho costo supera casi diez veces los costos del tratamiento por CCU, por lo cual dicha posibilidad se ve poco viable ${ }^{6}$.

El objetivo de esta investigación es estimar la DAP de los padres por vacunas para sus hijas anti VPH en Santiago de Chile. Adicionalmente, se busca identificar algunos de los factores que afectan la probabilidad de pago por dichas vacunas, lo que se realiza mediante el método de valoración contingente (MVC).

\section{Materiales y Métodos}

\section{Diseño del estudio}

Este estudio estima un modelo explicativo de la DAP por una vacuna anti VPH, basado en el método de valoración contingente. Este método es ampliamente utilizado, y utiliza escenarios hipotéticos, para revelar la DAP de los individuos, por evitar un daño, o por un mejoramiento, en la calidad de vida o ambiental.

La población objetivo corresponde a padres y madres pertenecientes al nivel $\mathrm{ABC} 1$ (ingreso familiar superior a \$CLP 1.300.000), C2 (con ingreso familiar entre 700.000 y 1.300.000), y C3 (ingreso familiar entre 400.000 y 700.000), residentes en la Región Metropolitana de Chile (RM).
Se decidió estudiar estos estratos por considerar que podrían disponer de los recursos económicos para adquirir las vacunas. Dada la imposibilidad de encuestar los hogares de dicha clase social, se realizaron encuestas por encuentro a dichos estratos.

La información utilizada en este estudio se obtuvo de la aplicación de dos instrumentos; una pre-encuesta y la encuesta final. Ambas encuestas fueron realizadas por los autores del estudio, entre el 20 de abril y el 4 de junio de 2011. Para ello se le entregó información a los entrevistados sobre el VPH y el CCU, indicándoles algunas estadísticas generales y los peligros de estos, luego se les preguntó la DAP por tratamientos, los que fueron basados en los atributos cercanos de las vacunas Cervarix ${ }^{\circledR}$ y Gardasi ${ }^{\circledR}$, las dos vacunas que están en el mercado para la prevención del VPH.

La encuesta final tenía tres secciones. La primera constaba de preguntas demográficas; la segunda parte contenía preguntas para determinar el conocimiento y el contacto con el VPH, en la tercera parte se le preguntaba sobre la DAP, previo a una introducción acerca del VPH y sus implicancias, donde los factores de riesgo asociados fueron basado en estudios previos ${ }^{4}$ y los costos en información obtenida de instituciones de salud previsional (Figura 1).

El virus del papiloma humano lo componen cerca de 200
tipos de virus, los cuales se contagian por contacto de
piel o relaciones sexuales. Ocho tipos de este virus son
responsables de $90 \%$ de los cánceres cervicales, y dos de
ellos son responsables de $70 \%$ de estos (16 y 18).
En Chile mueren dos (2) mujeres diariamente a causa de
este cáncer.
El virus del papiloma humano es el responsable de la ma-
yoría de los casos de lesiones genitales (herpes, verrugas
y sarpullidos).
Una vez diagnosticado, el costo total de un tratamiento
por un cáncer cervicouterino puede llegar a $\$ 3.500 .000$
anuales, además de las secuelas físicas y psicológicas de
la enfermedad.
Existe un tratamiento preventivo, el cual consiste en 3
vacunas, la cual previene el contagio de algunos virus del
papiloma humano, aunque este tratamiento, en algunos
casos, puede provocar reacciones alérgicas y un poco de
sensibilidad en la zona donde se aplica la vacuna.

Figura 1. Escenario contingente (hipotético) presentado a entrevistados 


\section{Análisis estadístico}

El modelo más usado para valorar escenarios hipotéticos en materia ambiental es el Modelo de Hanemann ${ }^{16}$, el que se puede expresar como $u_{j}=v_{j}\left(P, Y ; q_{j}\right)+\varepsilon_{j}$; donde: $j=0$ para la situación inicial, $j=1$ para la situación final (con el cambio de escenario hipotético predefinido), $P$ es el precio de los bienes o servicios, $Y$ es el ingreso del individuo, $q$ es el indicador de salud y características socioeconómicas de los individuos, y $\varepsilon_{j}$ es error aleatorio ${ }^{17}$. Los individuos pueden optar un escenario con y sin vacunas, es decir, asumen las consecuencias o pagan el costo por vacunarse $b_{t}$, donde $t$ indica los distintos costos que se les presentan a diferentes individuos, denominados vectores de pago. A partir de las respuestas de los individuos a la cantidad ofrecida $b_{t}$, se puede obtener la probabilidad de obtener una respuesta positiva por parte del individuo como $\operatorname{Pr}(S I)=\operatorname{Pr}\left[v_{1}\left(P, Y-b_{t} ; q_{1}\right)+\varepsilon_{1}>v_{0}\left(P, Y ; q_{0}\right)+\varepsilon_{0}\right]$, donde: $\eta \equiv \varepsilon_{0}-\varepsilon_{1}$. Por lo que: $\Delta v \equiv v_{1}\left(P, Y-b_{t} ; q_{1}\right)-v_{0}\left(P, Y ; q_{0}\right)$. Entonces, $\operatorname{Pr}(S I)=\operatorname{Pr}(\Delta v>\eta)=F_{\eta}(\Delta v)$. Donde $F_{\eta}$ es la función de distribución de $\eta$ que puede asumirse como función logística, expresada como $G(b)=\left[1+e^{\Delta v}\right]^{-1}$.

En general, las respuestas discretas son obtenidas con el formato dicotómico simple, donde los individuos responden "sí" o "no" al escenario hipotético con y sin vacuna. El método dicotómico doble utilizado en este estudio, consiste en agregar una segunda pregunta también de naturaleza dicotómica, con un valor superior, si dice a la primera pregunta dicotómica "sî", y uno inferior, si dice a la primera pregunta dicotómica "no" (Tabla 3). El modelo dicotómico doble provee una ganancia en la precisión de la matriz de varianza-covarianza de los coeficientes estimados, resultando en intervalos de confianza más pequeños ${ }^{18,19}$. La estimación se realiza utilizando el método de máxima verosimilitud.

De acuerdo a la definición de la diferencia de entre dos funciones indirectas de utilidad lineales $\left(\Delta V=\alpha-\beta b_{t}\right)$, la media de la DAP se puede estimar por medio de la esperanza de $C$, que se expresa como $E(C)=C^{*}=\alpha / \beta$. Un modelo expandido al anterior fue estimado en este estudio ${ }^{21}$, que se expresa como $\Delta V=\alpha_{1}+\beta b_{t}+\beta_{1} x_{1}+\beta_{2} x_{2}+\cdots \beta_{n} x_{n}$, donde $x_{i}$ es cualquier variable explicando la DAP de los padres, y $b_{t}$ refleja el costo de la vacuna. La DAP media por la vacuna se estima como $C=\alpha / \beta$, donde, $\Delta V=\alpha_{1}+\beta b_{t}+\beta_{1} x_{1}+\beta_{2} x_{2}+\cdots \beta_{n} x_{n}$, lo que
Tabla 1. Descripción de la muestra

\begin{tabular}{|lr|}
\hline Variable & Porcentaje (\%) \\
Número hijas & \\
$\quad$ Una & 95,3 \\
$\quad$ Dos o más & 4,7 \\
Edad hijas & \\
12-13 & 22,0 \\
14-15 & 50,0 \\
16-18 & 18,0 \\
Nivel de educación & \\
Básica, media completa & 66,8 \\
Universitaria incompleta & 14,5 \\
Superior completa y postgrado & 17,9 \\
Ingreso familiar & \\
Entre 1.300.000 y más (ABC1) & 45,6 \\
Entre 700.000 y 1.300.000 (C2) & 35,2 \\
Entre 400.000 y 700.000 (C3) & 19,2 \\
\hline
\end{tabular}

Tabla 2. Escenarios de costos de vacunas anti virus papiloma humano, pesos chilenos

\begin{tabular}{|cccc|}
\hline $\begin{array}{c}\text { Costo } \\
\text { inicial* }^{*}\end{array}$ & $\begin{array}{c}\text { Costo } \\
\text { superior** }^{* *}\end{array}$ & $\begin{array}{c}\text { Costo } \\
\text { inferior*** }^{* *}\end{array}$ & $\begin{array}{c}\text { Muestra } \\
\text { por costos }\end{array}$ \\
\hline 135.671 & 203.507 & 67.836 & 23 \\
181.536 & 272.304 & 90.768 & 51 \\
\hline 211.590 & 317.385 & 105.795 & 42 \\
235.740 & 353.610 & 117.870 & 40 \\
\hline 257.455 & 386.183 & 128.728 & 36 \\
\hline 278.399 & 417.599 & 139.200 & 38 \\
\hline 300.070 & 450.105 & 150.035 & 40 \\
\hline 324.263 & 486.395 & 162.132 & 42 \\
\hline 354.318 & 531.477 & 177.159 & 51 \\
\hline 400.183 & 600.275 & 200.092 & 23 \\
\hline
\end{tabular}

*Vector de pago (precios) o costos estimados usando rutina Dweabs, Cooper (1994) a partir de datos pre-encuesta, ${ }^{* *}$ costo presentado al entrevistado si responde si a costo inicial, ${ }^{* * *} \cos -$ to presentado al entrevistado si responde no a costo inicial.

Tabla 3. Respuestas sobre disposición a pagar determinados costos por vacuna anti virus papiloma humano

\begin{tabular}{|lc|}
\hline Variable & $\%$ \\
\hline Respuestas Sí-sí & 45,3 \\
\hline Respuestas Sí-NO & 30,1 \\
\hline Respuestas NO-SÍ & 20,5 \\
\hline Respuestas NO-NO & 4,1 \\
\hline
\end{tabular}


permite la consistencia con el modelo lineal de una función de diferencias de utilidad. Dado que la DAP media es la razón de dos coeficientes estimados, los intervalos de confianza son construidos en base a iteraciones siguiendo el método Krinsky y Robb ${ }^{20}$.

La muestra para el desarrollo de este estudio fue estimada usando un nivel de confianza de $95 \%$, un intevalo de confianza de 4,99\%, asumiendo varianza máxima, lo que dio un valor de 386 individuos a entrevistar.

\section{Resultados}

\section{Análisis descriptivo}

La encuesta final se aplicó a 386 personas de estratos ABC1, C2 y C3 de la Región Metropolitana de Chile, del total de personas entrevistadas, 95\% de los padres tenían 1 hija entre 12 y 18 años, donde las menores de 14 años eran las más representativas con $31 \%$ del total, de estas sólo 1\% de las menores se había realizado el Papanicolaou. El 74\% indicó conocer el VPH, pero ninguna de las personas entrevistadas había vacunado a sus hijas, de ellos $46,1 \%$ se enteró a través de la televisión, 20,2\% lo hizo por medio de amigos o familiares, $3,4 \%$ por el médico, el resto lo supo en su trabajo u otro medio. Respecto al conocimiento de las lesiones genitales (LG) y CCU, 75,1\% y 73,8\% de los entrevistados dijo conocer sobre las LG y el CCU, respectivamente. Además, $1 \%$ conocía a alguien con el VPH, $10 \%$ tiene a un conocido con LG y $2 \%$ con CCU. De los encuestados $6 \%$ supo sobre el virus hacía menos de dos meses, 39\% tomó conocimiento desde 2 a 12 meses antes, mientas que el resto lo conocía hacía más de 12 meses (Tabla 1).

Los entrevistados mostraron una actitud positiva a pagar por un tratamiento preventivo anti VPH. Sin embargo, una vez que se le mostraron potenciales costos de dichas vacunas en formato dicotómico doble (Tabla 2), 25\% de ellos no estaban dispuestos a pagar por este tratamiento, debido a que según ellos existían métodos más económicos para prevenir el contagio o que el tratamiento debería ser subvencionado por el Estado. No obstante, dentro de este grupo, 21\% estaría DAP a un precio 50\% menor al enfrentado en la entrevista, quedando sólo 4\%, que no estaría DAP a cualquier precio (Tabla 3). Los datos muestran que a mayor nivel de ingreso había una mayor dis- posición a pagar por una vacuna o tratamiento. Es así como $51 \%$ de los padres que poseen un ingreso sobre 1.300 .000 pesos, estaban dispuestos a pagar por una vacuna preventiva, en comparación con el 39\% de los padres que tienen un ingreso entre 400.000 a 700.000 pesos. Adicionalmente, como era de esperar, las personas con más integrantes mostraron una menor disposición a pagar por una vacuna.

Adicionalmente, se demostró una alta preocupación de los padres por el posible contagio de sus hijas por VPH. En una escala con niveles de 1 a 7 , siendo 7 el mayor grado de importancia y 1 el menor, 97\% marcó la opción 7 . En el caso de las LG se obtuvo $83 \%$ para el nivel más alto de importancia, $17 \%$ para el segundo nivel más alto de importancia. Con respecto al CCU, 92\% de los padres demostraron el nivel máximo de preocupación (nivel 7) y $8 \%$ el segundo nivel superior de la escala (nivel 6).

\section{Modelo econométrico}

El modelo final se estima usando la rutina del Programa $\mathrm{VCM}^{21}$ quedando de la siguiente forma:

$$
\Delta v_{j}=\alpha_{1}+\beta_{1} b_{t}+\beta_{2} G F+\beta_{3} I N+\beta_{4} C O+\eta
$$

donde $b_{t}=$ costos vacuna, $G F$ es el número de personas que componen el grupo familiar, $I N$ es el ingreso familiar mensual, $C O$ es la variable dicotómica sobre si posee o no conocimiento sobre el VPH. Los resultados del modelo se presentan en la Tabla 4, cuya expresión funcional es:

$$
\begin{gathered}
\Delta v_{j}=5,1451+(1,1564044 \mathrm{e}-05) b_{t}+0,5472 G F+ \\
0,5634 I N+0,6469 C O
\end{gathered}
$$

Todos los coeficientes son significativos con un valor-p igual a cero. El coeficiente de costos de las vacunas tiene signo negativo, lo que concuerda con la teoría económica, que a mayor costo menor es la probabilidad de adquirir la vacuna. La variable tamaño del grupo familiar con signo negativo significa que sí aumenta el tamaño de la familia se reduce la probabilidad de adquirir las vacunas preventivas. Otra variable que incide es el ingreso (IN) donde el signo positivo indica que a mayor ingreso mayor es la probabilidad de pagar por una vacuna. La variable conocimiento también es relevante e implica que a mayor conocimiento sobre VPH los padres aumentan la probabilidad de pagar por una vacuna. 
Tabla 4. Modelo econométrico logístico

\begin{tabular}{|lccr|}
\hline Nombre variable & Variable & Coeficiente & t-value \\
\hline Constante & $\alpha_{1}$ & 5,1451 & $8,364^{*}$ \\
Costo vacuna & $b_{t}$ & $-1,1564044 \mathrm{e}-05$ & $-15,870^{*}$ \\
\hline Tamaño grupo familiar & GF & $-0,5472$ & $-4,023^{*}$ \\
\hline Ingreso & IN & 0,5634 & $3,823^{*}$ \\
Conocimiento vph & CO & 0,6469 & $2,746^{*}$ \\
log likelihood & $-417,15$ & & \\
Muestra & 386 & & \\
\hline
\end{tabular}

${ }^{*}$ p-value $<0,0000$, todos los coeficientes significtaivos al $99 \%$ de confianza.

Del modelo estimado se puede obtener la DAP media de los padres por vacunar a sus hijas, cuyo monto alcanza a 757,99 dólares americanos (USD), (378.743 pesos chilenos), cuyo intervalo al 99\% de nivel de confianza entrega valores entre 716 y 810 USD, considerando un tipo de cambio 499,67 CLP por USD al 13 de noviembre de 2011. Estos valores representan el beneficio económico privado del escenario de vacunarse con respecto al de no vacunarse.

\section{Discusión}

La disponibilidad de vacunas anti VPH se espera que resulte en una mayor prevención de los jóvenes contra este virus, aunque ello depende de una serie de factores, donde uno de los factores es la disposición de los padres a vacunar a sus hijas. Similar a otros estudios ${ }^{22}$, este trabajo muestra una alta disposición de los padres a vacunar sus hijas contra el VPH, a pesar que ninguna de las personas entrevistadas las había vacunado, porque su decisión final respecto a elegir un determinado escenario está determinada por el costo de las vacunas o tratamiento y por su restricción de presupuestaria.

Lo anterior se evidencia cuando se enfrentó a los padres con determinados precios, donde cerca de $25 \%$ no estaban DAP por una vacuna, por considerar que existían otros métodos de prevención más baratos o que el Estado debiera proveerlas. Lo anterior, puede ser un reflejo de lo que ocurre en la realidad, ya que los médicos, a pesar de recetar las inyecciones, estas no siempre son aplicadas, debido a la restricción de ingresos de los padres.

El rechazo sería mayor si se incorporan los estratos socioeconómicos más bajos de la población. Algunos autores mencionan que la vacunación de adolescentes en Chile podría ser costo-ahorrativa, y que sería adecuado una vacunación universal ${ }^{1}$. Sin embargo, un estudio para Chile ${ }^{6}$ muestra que los costos de vacunar son más altos que tratar un CCU.

Uno de los escasos estudios que considera el factor precio y la DAP por una vacuna que reduzca el riesgo a contraer el VPH en Estados Unidos de Norteamérica alcanza valores entre 560 y 660 USD $^{13}$. Estos valores son casi 15\% menores que los que están DAP los padres de familias de Chile. En parte, la diferencia puede explicarse porque la población considerada en la RM era de estratos de ingresos altos a diferencia del caso de Estados Unidos de Norteamérica. Además, nuestro estudio permitió incluir más variables determinantes de la conducta de compra de los padres.

Las DAP estimadas por vacunas en este estudio son superiores a aquellos realizados vía análisis costo-efectividad ${ }^{14,15}$. Además, los precios de mercado en Chile de las vacunas, que varían por el tipo de vacuna y el vacunatorio, por las tres dosis, están entre 360 a 500 USD, montos que son menores a los beneficios privados estimados por vacunarse (758 USD). Este estudio es el primero en Chile que cuantifica los beneficios económicos privados de la vacunación anti VPH, información reconocida como altamente valorable en el diseño de políticas públicas en salud ${ }^{13,23}$.

\section{Referencias}

1. Parkin DM, Bray F. The burden of HPV-related cancer. In Vaccine 2006; 24: 511-25 (Chapter 2).

2. Muñoz N. Human papillomavirus and cancer: the epidemiological evidence. Journal of Clinical Virology, 2000; 19 (1): 1-5.

3. Wieland U, Pfister, H. 1997. Papillomavirus in human pathology: Epidemiology, pathogenesis and oncogenic role. Capítulo 1, en: Human Papilloma Virus Infection. Editado por: Gross, G., Barrasso, R. Alemania: Editorial Ullstein Mosby, 1-16.

4. Abarca K, Valenzuela MT, Vergara R, Luchsinger V, Muñoz A, Jiménez de la Jara J, et al. Comité Consultivo de Inmunizaciones, So- 
ciedad Chilena de Infectología. Declaración del Comité Consultivo de Inmunizaciones de la SOCHINF respecto a la vacuna antivirus papiloma humano, septiembre 2008. Rev Med Chile 2008; 136: 1485-92.

5. MINSAL. 2008. Manual de Manejo y Tratamiento de Infecciones de Transmisión Sexual (ITS)'. Norma General Técnica No 103, aprobada por Decreto Exento No 424 del 17 de julio de 2008. República de Chile.

6. MINSAL. 2011. Sistematización de la Información sobre Cáncer Cérvico Uterino en Chile: Revisión y Análisis de Estudios de Costo-efectividad de la Vacuna contra VPH. ISBN: 978-956-8823-96-2 Registro Propiedad Intelectual: 202.390. Febrero.

7. Dunne E, Nielson C, Stone K, Markowitz L, Giuliano R. Prevalence of HPV infection among men: A systematicreview of the literature. J Infect Dis 2006; 194: 1044-57.

8. Muñoz N, Castellsagué X, Berrington A, Gissmann L. Chapter 1: VPH in the etiology of human cancer. In: Bosch FX, Cuzik J, Schiller JT, Garnett GP, Micheus A, Franco EL and Wright TC, editors. VPH vaccines and screening in the prevention of cervical cancer. Vaccine 2006; 24 (Suppl 3): S3/1-10.

9. Zimet GD, Mays RM, Sturm L, Ravert A, Perkins S, Juliars B. Parental attitudes about sexually transmitted infection vaccination for their adolescent children. (Reprinted) Arch Adolesc Med 2005; 159: 132-37.

10. Lazcano-Ponce E, Herrero R, Muñoz N, HernándezÁvila M, Salmeron J, Leyva A, et al. High prevalenceof human papillomavirus infection in Mexican males: comparative study of penile-urethral swabs and urine samples. Sex Transm Dis 2001; 28: 277-80.

11. Davis K, Dickman ED, Ferris D, Dias JK. Human papillomavirus vaccine acceptability among parents of $10-$ to 15-year-old adolescents. J Lower Gen Tract Dis 2004; 8: 188-94.

12. Zimet GD, Mays RM, Winston Y, Kee R, Dickes J, Su L. Acceptability of human papillomavirus immunization. J Womens Health Gend Based Med 2000; 9: 47-50.

13. Brown D, Johnson Fr, Poulos C, Messonnier M. Mo- thers' preferences and willingness to pay for vaccinating daughters against human papillomavirus. Vaccine 2010; 28: 1702-8.

14. Jimm, Choi YH, Edmunds WJ. Economics evaluation of human papillomavirus vaccination in the United Kingdom. BMJ2 008; 377: a769.

15. Brisson M, Van De Velden, De Wals P, Boily M-C. The potential cost-effectiveness of prophylactic human papillomavirus vaccines in Canadá. Vaccine 2007; 25 (29): 5399-408.

16. Hanemann M. Welfare evaluation in contingent valuation experiments with discrete responses. American Journal of Agricultural Economics 1984; 66: 222-41.

17. Vásquez F, Cerda A, Orrego S. Valoración económica del ambiente. Thomson Learning. Buenos Aires, Argentina; 2007.

18. Hanemann M, Loomis J, Kanninen B. Statistical efficiency of double-bounded dichotomous choice contingent valuation. American Agricultural Economics 1991; 73 (1): 1255-63.

19. Cooper J. Optimal bid selection for dichotomous choice contingent valuation surveys. Journal of Environmental Economics and Management 1993; 24: 25-40.

20. Park T, Loomis JB, Creel. Confidence intervals for evaluating benefit estimates from dichotomous choice contingent valuation studies. Land Economics 1991; 67: 64-73.

21. Cooper J, Hellerstein D. CVM A Collection of Dichotomous Choice and Double-Bounded CVM Related Routines. USDA/NRS/Resources and Technology Division, 1994, 1301 New York. Ave. NW, Rm. 508, Washington DC, 20005-4788_1994.

22. Mays RM, Sturm LA, Zimet GD. Parental perspectives on vaccinating children against sexually transmitted infections. Soc Sci Med 2004; 58: 1405-13.

23. Wordsworth S, Ryan M, Waugh N. Cost and benefits of cervica screening IV: valuation by women of the cervical screening programme. Cytopathology 2001; 12 (6): 36776. 